\title{
Curability of laparoscopic gastrectomy for gastric cancer: an analysis of 10 years' experience
}

\author{
Xiaogiao Zhang, Nobuhiko Tanigawa, Eiji Nomura, and Sang-Woong Lee \\ Department of General and Gastroenterological Surgery, Osaka Medical College, 2-7, Daigaku-machi, Takatsuki, Osaka 569-8686, Japan
}

\begin{abstract}
Background. Laparoscopic gastrectomy is becoming widely used for the management of gastric cancer. To evaluate its oncologic feasibility, we analyzed the curability of laparoscopic gastrectomy based on our 10-year experience.

Methods. All laparoscopic gastrectomies for gastric cancer performed in the past 10 years, with the exception of those converted to open surgery, were evaluated. The number of dissected lymph nodes and the proximal and distal distances between the primary lesion and resection lines were analyzed and compared among different procedures. Laparoscopic and open $\mathrm{D} 2$ resection were also compared.

Results. Most of the 391 eligible patients fulfilled the oncologic requirement of current treatment guidelines. The mean proximal and distal distances were $3.73 \pm 2.11 \mathrm{~cm}$ and $5.31 \pm$ $3.26 \mathrm{~cm}$, respectively. A distance of less than $1 \mathrm{~cm}$ occurred in only 10 patients proximally and 5 patients distally, with pathological examination results being negative. In each operation, an average of 22 lymph nodes were dissected $(21.7 \pm 12.1)$. Laparoscopic D2 resection possessed the same capacity as open surgery in terms of lymph node dissection. The proximal distance in open surgery was about $1 \mathrm{~cm}$ longer than that in laparoscopic gastrectomy $(4.99 \pm 2.59 \mathrm{~cm}$ vs $4.06 \pm 1.87 \mathrm{~cm}$; $P=0.038)$, while the difference between distal distances was not significant $(6.94 \pm 3.52 \mathrm{~cm}$ vs $7.24 \pm 4.64 \mathrm{~cm} ; P=0.187)$.

Conclusion. From the point of view of curability, laparoscopic operation is an oncologically safe procedure for the management of gastric cancer, at least for stage I and II disease.
\end{abstract}

Key words Stomach neoplasms · Gastrectomy · Laparoscopy

\section{Introduction}

Laparoscopic gastrectomy has been applied in the surgical management of gastric cancer in recent decades [1],

Offprint requests to: $\mathrm{N}$. Tanigawa

Received: March 13, 2008 / Accepted: July 2, 2008 and its application is increasing. In Asian countries, such as Japan and Korea, it has become a standard therapy for early-stage gastric cancer [2,3], but controversy remains about the feasibility of laparoscopic radical gastrectomy for cancer of the stomach, and one of the most important questions is about the curability of laparoscopic gastrectomy. Laparoscopic gastrectomy has been performed at our institution for gastric cancer since 1998. Here we analyze our 10-year experience, and compare the laparoscopic procedure with open surgery to evaluate its curability and feasibility for gastric cancer.

\section{Patients and methods}

\section{Patients and operative procedures}

All gastric cancer patients treated with laparoscopic surgery from January 1998 to December 2007 were enrolled. Patients who received only laparoscopic exploration and those with conversion to laparotomy were excluded.

All patients underwent surgery under general anesthesia in a supine position with legs apart. The operation was performed using the five-port technique. The pressure of $\mathrm{CO}_{2}$ in the pneumoperitoneum was 8$10 \mathrm{mmHg}$. Mobilization of the stomach and dissection of the perigastric lymph nodes were performed following the Japanese Gastric Cancer Association (JGCA) Treatment guidelines for gastric cancer in Japan [4]. The range of gastric resection and extent of lymphatic dissection were determined according to the location of the primary lesion and the clinical stage. The types of gastric resection included mucosectomy, wedge resection, segmental gastrectomy, laparoscopic (assisted) pyloric-preserving gastrectomy (LPPG), laparoscopic (assisted) distal gastrectomy (LDG), laparoscopic (assisted) proximal gastrectomy (LPG), and laparo- 
scopic (assisted) total gastrectomy (LTG). Lymphatic dissection included D0, which means no lymphatic dissection or incomplete dissection of group 1 lymph nodes; $\mathrm{D} 1$, dissection of group 1 lymph nodes; D1 $+\alpha$, dissection of group 1 lymph nodes plus no. 7 and no. 8 a lymph nodes if the primary focus is located in the lower third of the stomach; D1 $+\beta$, dissection of group 1 lymph nodes plus no. 7, no. 8a, and no. 9 lymph nodes; and D2, which refers to the dissection of all group 1 and 2 lymph nodes. In some cases, lymphadenectomy extending between D1 and D2 was defined as selective D2 (sD2). For each operation, the curative potential was evaluated by the operator as resection $\mathrm{A}, \mathrm{B}$, or $\mathrm{C}$ according to the JGCA Japanese classification of gastric carcinoma [5].

\section{Variables}

General and clinicopathological data of eligible patients were retrieved from medical reports and reviewed retrospectively. The variables included sex, age, pathological stage according to the Japanese classification of gastric carcinoma [5], operative procedures and the extent of lymphatic dissection, the number of dissected lymph nodes and those with metastasis, the status of the specimen margin, and the distances from the lesion to the proximal and distal margins (proximal distance and distal distance). After endoscopic mucosal resection (EMR), the distance was measured from the scar margin following EMR.

\section{Evaluation of curability}

According to the Japanese classification of gastric carcinoma of the JGCA and the Gastric cancer clinical practice guidelines of the National Comprehensive Cancer Network (NCCN), the criteria for the extent of a possibly curable gastric cancer operation included: no involvement of the proximal and distal margins, with no less than 10-mm proximal and distal distances; and sufficient lymph node dissection, with no fewer than 15 lymph nodes dissected [6]. All eligible patients were evaluated using these criteria. Curability-related variables were compared among different operative procedures and lymph node dissections.

\section{Comparison of curability between laparoscopic and open radical gastrectomy with $D 2$ dissection}

To compare the curability of surgery between laparoscopic and open radical gastrectomy, procedures with D2 dissection were evaluated. All patients with laparoscopic gastrectomy with D2 dissection were enrolled. Patients with open radical gastrectomy (resection A or B) with D2 dissection in the most recent 2 years were selected as the control group, excluding patients with combined splenectomy. The number of dissected lymph nodes, and the proximal and distal distances were compared.

\section{Statistics}

Values for all continuous variables were expressed as means \pm SD. Student's $t$-test, one-way analysis of variance (ANOVA), and Dunnett's $C$ test were used for comparison and post-hoc multiple comparison of means. The $\chi^{2}$ test (Pearson's $\chi^{2}$ test) was used for analysis of categorical variables. $P<0.05$ was considered to be significant, and all statistical analysis was performed with SPSS13.0 software (SPSS, Chicago, IL, USA).

The planned sample size for the comparison of D2 dissection between laparoscopic operation and the open control group was 31. This was calculated on the basis that an average of $30.7 \pm 11.1$ lymph nodes had been dissected by D2 dissection with open surgery in the most recent 2 years, with the hypothesis of about $25 \%$ reduction (8 lymph nodes, which mainly referred to incomplete or no dissection of stations no. 11p, 12a, and $14 \mathrm{v})$ in the number of lymph nodes dissected laparoscopically, and a statistical power of $80 \%$ for a twotailed type-I error of $5 \%$.

\section{Results}

\section{Patient demographics}

From January1998 to December 2007, 1441 patients with gastric cancer were treated surgically at our institution. Among them, 404 patients, 271 men $(67.1 \%)$ and $133(32.9 \%)$ women, were treated with laparoscopic surgery. Their average age was $63.3 \pm 10.6$ years (range, 32-92 years). Conversion to open surgery occurred in 13 patients and they were excluded from the evaluation of curability. The pathological stage, operation procedure, and range of lymph node dissection for the remaining 391 patients are shown in Table 1. Most of the patients were stage I, and LDG was the most common procedure. More than $80 \%$ of the patients received sub$\mathrm{D} 2$ dissection ( $\mathrm{D} 1+\alpha, \mathrm{D} 1+\beta$, or $\mathrm{sD} 2)$. The 18 operations with D0 dissections were all local resections, except for 1 case of segmental resection. Except for 2 cases of resection $\mathrm{C}$ and 8 cases of resection $\mathrm{B}$, the remaining $381(97.4 \%)$ cases were all classified as resection $\mathrm{A}$.

During the 1- to 113-month follow-up period (median, 40 months), no port-site recurrence was observed. Four patients relapsed postoperatively; peritoneal metastasis occurred in three patients and liver metastasis in one patient. 
Table 1. Clinicopathological characteristics of patients $(n=391)$

\begin{tabular}{lc}
\hline Variable & $\begin{array}{c}\text { Number of } \\
\text { patients }(\%)\end{array}$ \\
\hline Pathological stage & \\
Stage I & $371(94.9)$ \\
$\quad$ Ia (T1N0M0) & $330(84.4)$ \\
Ib & $41(10.5)$ \\
Stage II & $17(4.3)$ \\
Stage IIIa (T3N1M0) & $2(0.5)$ \\
Stage IV (T3N3M0, T3N2P1) & $1(0.3)$ \\
Operation procedure & \\
Mucosectomy & $2(0.5)$ \\
Wedge resection & $22(5.4)$ \\
Segmental resection & $17(4.3)$ \\
LPPG & $129(33)$ \\
LDG & $170(43.5)$ \\
LPG & $40(10.2)$ \\
LTG & $11(2.8)$ \\
Range of lymph node dissection & \\
D0 & $18(4.6)$ \\
D1 & $16(4.1)$ \\
D1 $+\alpha$ & $111(28.4)$ \\
D1 $+\beta$ & $124(31.7)$ \\
sD2 & $79(20.2)$ \\
D2 & $43(11.0)$ \\
\hline
\end{tabular}

LPPG, laparoscopic (assisted) pyloric-preserving gastrectomy; LDG, laparoscopic (assisted) distal gastrectomy; LPG, laparoscopic (assisted) proximal gastrectomy; LTG, laparoscopic (assisted) total gastrectomy; sD2, selective D2

\section{Comparison of margin distances among procedures}

The mean proximal and distal distances were $3.73 \pm$ $2.11 \mathrm{~cm}$ (range, 0 to $14.0 \mathrm{~cm}$ ) and $5.31 \pm 3.26 \mathrm{~cm}$ (range, 0.2 to $19.0 \mathrm{~cm}$ ), respectively. In 10 patients, the proximal distance was less than $1.0 \mathrm{~cm}$. Of these 10 patients, 5 had wedge resections ( 5 of 22 cases of wedge resection); the other 5 patients included 2 patients with mucosectomy (2/2), 1 patient with LPPG (1/129), and 2 patients with LDG (2/170). The 1 patient with 0 -cm proximal distance had undergone LDG following EMR and the resection line was located on the scar margin of the endoscopic resection. All specimens with a proximal distance of less than $1 \mathrm{~cm}$ were negative on pathological examination. In only 1 patient, the proximal margin after LPPG was positive, in spite of a $2.3-\mathrm{cm}$ proximal distance and, for this reason, total gastrectomy was performed later. All distal margins were negative on pathological examination. Only in 5 patients was the distal distance less than $1.0 \mathrm{~cm}$; 1 patient had had a mucosectomy (1/2) and the others had all had wedge resections (4/22). The differences for percentages of distances of less than $1-\mathrm{cm}$ among the different kinds of procedure were statistically significant $(P<0.001$ for both proximal distance and distal distance). Further intraoperative resection to achieve a negative margin was performed in 12 of the
391 patients; 9 of these were performed because of an insufficient proximal distance.

The differences between the proximal and distal distances among the different procedures were statistically significant $(\mathrm{F}=10.39$ and 16.36 for proximal and distal distances, respectively;, $P<0.001)$. The results of posthoc multiple comparisons are shown in Fig. 1.

\section{Comparisons of different lymph node dissections}

In each operation, an average of 22 lymph nodes were dissected $(21.7 \pm 12.1)$; the number ranged from 0 to 84 . Lymphatic metastasis occurred in 33 patients (33/391; $8.4 \%)$, and in most of these patients $(29 / 33 ; 87.9 \%)$, the number of affected lymph nodes was less than 6 . In only 1 patient, metastasis was detected in more than 15 lymph nodes (18 of 68 retrieved lymph nodes). In 100 patients $(100 / 391 ; 25.6 \%)$, the number of lymph nodes retrieved was less than 15 . Among these patients, lymphatic metastasis was detected in 11 lymph nodes. These cancer-affected lymph nodes belonged to 6 patients. For each of these 6 patients, more than 10 lymph nodes were dissected and fewer than $20 \%$ of the nodes were affected by cancer.

The difference among numbers of retrieved lymph nodes by different lymph node dissections was statistically significant $(\mathrm{F}=26.50 ; P<0.001)$; the results of post-hoc multiple comparisons are shown in Fig. 2.

\section{Comparison between open and laparoscopic D2 dissection}

To compare curability-related variables between open and laparoscopic D2 dissection, all 43 patients with laparoscopic D2 dissection were selected. The control group was composed of all 57 patients with open radical gastrectomy with D2 dissection performed in 2006 and 2007, excluding those with combined splenectomy. Distal gastrectomy was the main procedure in both groups ( $38 / 43$ vs $50 / 57 ; P=0.921)$ and the groups were homogeneous except for the pathological stage of disease, as most patients in the control group were affected with advanced cancer. For the extent of gastric resection, the proximal distance in open surgery was about $1 \mathrm{~cm}$ longer than that in laparoscopic gastrectomy (open vs laparoscopic, $4.99 \pm 2.59 \mathrm{~cm}$ vs $4.06 \pm 1.87 \mathrm{~cm}$; $P=0.038$ ), while the difference between distal distances was not significant $(6.94 \pm 3.52 \mathrm{~cm}$ vs $7.24 \pm 4.64 \mathrm{~cm}$; $P=0.187$ ). The numbers of dissected lymph nodes were similar in the two groups (open vs laparoscopic, $30.70 \pm$ 11.09 vs $33.18 \pm 15.03 ; P=0.156$ ), but the mean number of cancer-affected lymph nodes was significantly higher in the open-surgery group $(2.89 \pm 3.55$ vs $0.86 \pm 2.98$; $P=0.04)$. 


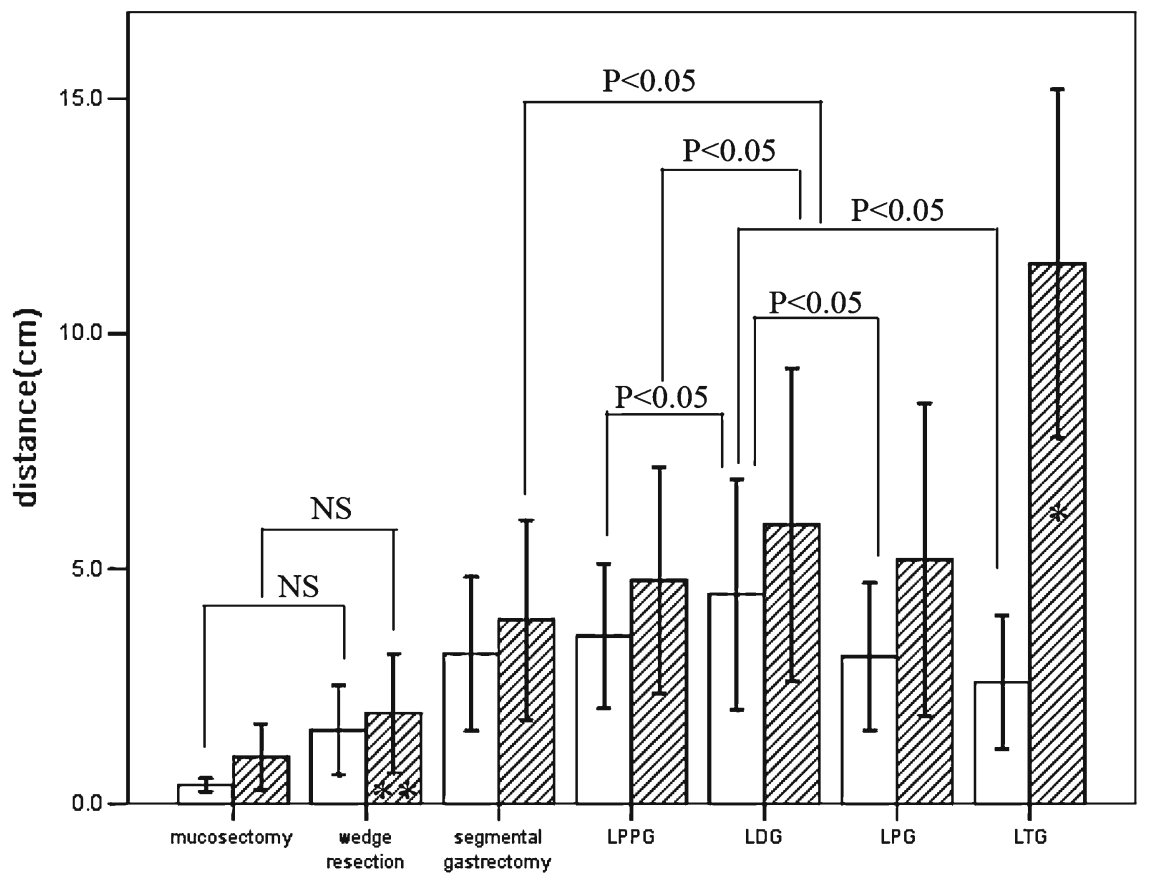

operation procedure

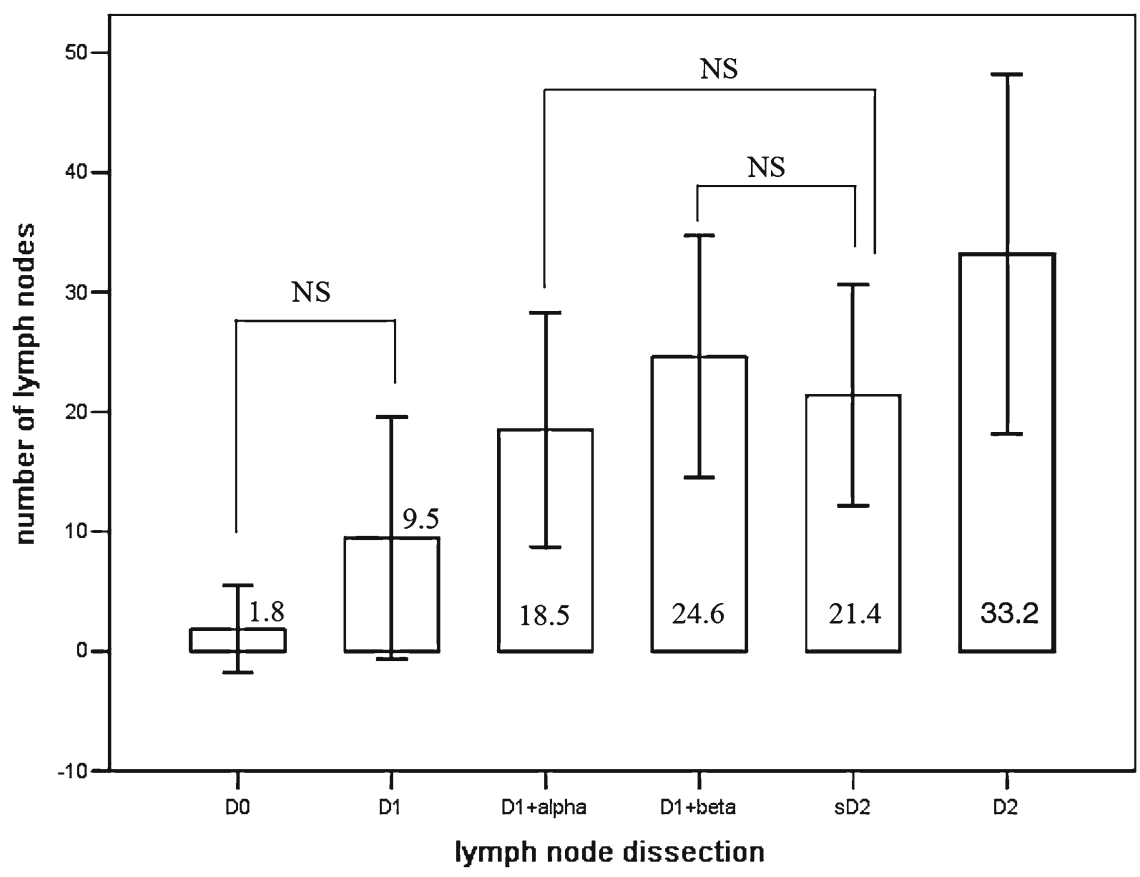

Fig. 1. Results of post-hoc multiple comparisons of proximal distance (in $\mathrm{cm}$; white bars) and distal distance (in $\mathrm{cm}$; hatched bars) among different procedures. Single asterisk, Distal distance in laparoscopic (assisted) total gastrectomy $(L T G)$ was significantly longer than that with the other six procedures. Double asterisk, Distal distance in wedge resection was significantly shorter than that in the other procedures, except for mucosectomy; the distal distance in laparoscopic (assisted) distal gastrectomy $(L D G)$ was longer than that in segmental gastrectomy and laparoscopic (assisted) pyloric-preserving gastrectomy $(L P P G)$; and the proximal distance in LDG was also significantly longer than that in LPPG, laparoscopic (assisted) proximal gastrectomy $(L P G)$, and LTG. NS, Not statistically significant. Error bars, \pm 1 SD

Fig. 2. Results of post-hoc multiple comparisons for numbers of retrieved lymph nodes according to different lymph node dissections. The differences in numbers of retrieved lymph nodes between the compared pairs of lymph node dissection types were statistically significant, with the exceptions of D0 vs D1, D1 + alpha vs sD2, and D1 + beta vs sD2. D0, No lymphatic dissection or incomplete dissection of group 1 lymph nodes; $D 1$, dissection of group 1 lymph nodes; $D 1+$ alpha, dissection of group 1 lymph nodes plus no. 7 and no. 8 a lymph nodes if the primary focus is located in the lower third of the stomach; D1 + beta, dissection of group 1 lymph nodes plus no. 7, no. 8a, and no. 9 lymph nodes; $s D 2$, selective D2, lymphadenectomy extending between D1 and D2; D2, dissection of all group 1 and 2 lymph nodes. NS, Not statistically significant. Error bars, $\pm 1 \mathrm{SD}$

\section{Discussion}

The application of laparoscopic techniques in the management of gastric cancer has increased significantly in recent years. In some Asian countries, such as Japan and Korea, laparoscopic gastrectomy has become the standard therapy for early-stage gastric cancer [2,3], but controversy remains about the suitability of laparo- scopic radical gastrectomy for cancer of the stomach. Some shortcomings of the laparoscopic procedure, such as the lack of direct palpation of the primary lesion and the difficulty of meticulous dissection, have led to controversy about the curability of laparoscopic surgery for gastric cancer, especially because of the relatively complicated clinical anatomy of radical gastrectomy. For this reason, in the present study, we examined the 
extent of gastric resection and lymphatic dissection as key indices for evaluating the curability and oncologic feasibility of laparoscopic gastrectomy for gastric cancer.

Of course, some studies have reported on the curability of laparoscopic gastrectomy and compared the curability with that in open surgery [7-18]. Most of these studies verified the feasibility of laparoscopic resection for gastric cancer, mainly in terms of the number of lymph nodes retrieved, and, in some cases, in terms of the distances between the primary lesion and specimen margins. On the other hand, perhaps due to the limited application and immature techniques of laparoscopic gastrectomy in the early period, the sample sizes in most of these studies were small or even insufficient, and usually only a given kind of procedure was evaluated. Since 1998, more than 400 cases of laparoscopic gastrectomy have been performed at our institution. Based on these data, we have reviewed our 10-year experience. We compared the different procedures of laparoscopic gastrectomy, and we also made comparisons between open and laparoscopic D2 lymphatic dissection, so as to obtain systematic insights into the curability of laparoscopic gastrectomy for the treatment of gastric cancer.

The difficulty in determining the exact location of the primary lesion in early gastric cancer is well known, due to the lack of direct palpation and the presence of lesions that are small and superficial. It is therefore important to mark the tumor with pigmentation or clips endoscopically before surgery. At the time of resection, the relationship between the mark and the lesion, and the distance from the resection line to the mark should be checked carefully, especially in the proximal resection in LDG and LPPG. In our series, the mean distances to both the distal and proximal margins were sufficient according to the JGCA gastric cancer treatment guidelines [4] and were comparable to those reported by other authors $[17,19,20]$, but in 15 patients $(3.8 \%)$, the proximal or distal distances were less than $1 \mathrm{~cm}$. Furthermore, additional resection was performed in 12 patients to achieve a negative margin or sufficient distance. When compared with the proximal distance in open surgery, that in laparoscopic gastrectomy was significantly shorter. Although a statistically significant 1 $\mathrm{cm}$ difference between $4 \mathrm{~cm}$ and $5 \mathrm{~cm}$ is not practically important in early gastric cancer, such a tendency of a shorter proximal distance should be noted, and attention should be paid to achieve a safe margin, especially for the proximal resection line [13]. As most of the insufficient distances in our study occurred in patients who had had mucosectomies and wedge resections, more attention should be paid to the selection of candidates for these two procedures, and attention should also focus on performing sufficient resection.
According to the NCCN [6] criteria, to evaluate the lymph node status, at least 15 lymph nodes should be retrieved. In our series, in about one-quarter of the patients, the number of lymph nodes dissected was less than 15 . But as there were only very few cases of lymphatic metastasis, and the proportion of cancer-affected lymph nodes was quite low, we think that, even in these patients, the lymphatic dissection was sufficient, either for therapy or for staging. When retrieved lymph node numbers were compared according to different extents of lymphatic dissection, significant differences were revealed between D0-D1 and subD2 $(\mathrm{D} 1+\alpha, \mathrm{D} 1+\beta$, sD2) dissections, and between D0-D1 and D2 dissections. These findings confirmed the exact differences among the different extents of dissection. Perhaps the three kinds of subD2 dissection have the same role in staging for early gastric cancer, as the numbers of dissected lymph nodes in these three kinds of dissection were not significantly different.

Compared with open surgery, it is relatively difficult to perform a standard D2 lymphadenectomy laparoscopically, mainly due to difficulties in dissecting stations no. $11 \mathrm{p}, 12 \mathrm{a}$, and $14 \mathrm{v}$. The results of many studies have confirmed the curability of laparoscopic surgery in terms of the numbers of dissected lymph nodes [8-12, 14-18]. However, a stratified analysis by Kim et al. [13] revealed that the number of lymph nodes retrieved by laparoscopic D2 dissection was significantly less than the number retrieved by open surgery, while the number of lymph nodes retrieved by $\mathrm{D} 1+\alpha$ or $\mathrm{D} 1+\beta$ lymphadenectomy was the same as that for open surgery. We consider that this difference was not a result of insufficient laparoscopic D2 lymphadenectomy, but of immature operative techniques, as the numbers of lymph nodes dissected by $\mathrm{D} 1+\beta$ and by $\mathrm{D} 2$ lymphadenectomy were almost the same in their study. The results of our series showed that the number of lymph nodes retrieved by laparoscopic D2 lymphadenectomy was not significantly different from the number retrieved by open D2 lymphadenectomy. On the other hand, the number of lymph nodes retrieved by laparoscopic D2 lymphadenectomy was significantly higher than the number retrieved by other laparoscopic lymphadenectomies. Based on this result, we suggest that laparoscopic surgery has the same capacity as open surgery in terms of lymph node dissection, at least to the extent of no more than D2 dissection.

Based on these results, we think that laparoscopic surgery is an oncologically safe procedure for the management of gastric cancer, at least for stage I and II disease. It is now possible to obtain an endpoint index earlier, such as the survival rate, according to the curability-related variables that we examined here, and the satisfactory survival of gastric cancer patients treated laparoscopically is anticipated in the near future. 
Acknowledgments This study was supported in part by the Japan-China Sasakawa Medical Fellowship.

\section{References}

1. Kitano S, Iso Y, Moriyama M, Sugimachi K. Laparoscopy-assisted Billroth I gastrectomy. Surg Laparosc Endosc 1994;4:146-8.

2. Kitano S, Shiraishi N, Uyama I, Sugihara K, Tanigawa N, Japanese Laparoscopic Surgery Study Group. A multicenter study on oncologic outcome of laparoscopic gastrectomy for early cancer in Japan. Ann Surg 2007;245:68-72.

3. Kim MC, Kim HH, Jung GJ. Surgical outcome of laparoscopyassisted gastrectomy with extraperigastric lymph node dissection for gastric cancer. Eur J Surg Oncol 2005;31:401-5.

4. Japanese Gastric Cancer Association. Treatment guidelines for gastric cancer in Japan (in Japanese). 2nd ed. Tokyo: Kanehara; 2004.

5. Japanese Gastric Cancer Association. Japanese classification of gastric carcinoma - 2nd English edition - Gastric Cancer 1998;1:10-24.

6. Ajani J, Bekaii-Saab T, D'Amico TA, Fuchs C, Gibson MK, Goldberg $\mathrm{M}$, et al. Gastric cancer clinical practice guidelines. J Natl Compr Canc Netw 2006;4:350-66.

7. Shimizu S, Uchiyama A, Mizumoto K, Morisaki T, Nakamura K, Shimura $\mathrm{H}$, et al. Laparoscopically assisted distal gastrectomy for early gastric cancer: is it superior to open surgery? Surg Endosc 2000;14:27-31.

8. Adachi Y, Shiraishi N, Shiromizu A, Bandoh T, Aramaki M, Kitano S. Laparoscopy-assisted Billroth I gastrectomy compared with conventional open gastrectomy. Arch Surg 2000;135:80610.

9. Reyes CD, Weber KJ, Gagner M, Divino CM. Laparoscopic vs open gastrectomy. A retrospective review. Surg Endosc 2001;15: 928-31

10. Yano H, Monden T, Kinuta M, Nakano Y, Tono T, Matsui S, et al. The usefulness of laparoscopy-assisted distal gastrectomy in comparison with that of open distal gastrectomy for early gastric cancer. Gastric Cancer 2001;4:93-7.

11. Lee JH, Han HS, Lee JH. A prospective randomized study comparing open vs laparoscopy-assisted distal gastrectomy in early gastric cancer: early results. Surg Endosc 2005;19:168-73.

12. Huscher CG, Mingoli A, Sgarzini G, Sansonetti A, Di Paola M, Recher A, et al. Laparoscopic versus open subtotal gastrectomy for distal gastric cancer: 5-year results of a randomized prospective trial. Ann Surg 2005;241:232-7.

13. Kim MC, Kim KH, Kim HH, Jung GJ. Comparison of laparoscopy-assisted by conventional open distal gastrectomy and extraperigastric lymph node dissection in early gastric cancer. J Surg Oncol 2005;91:90-4.

14. Hayashi H, Ochiai T, Shimada H, Gunji Y. Prospective randomized study of open versus laparoscopy-assisted distal gastrectomy with extraperigastric lymph node dissection for early gastric cancer. Surg Endosc 2005;19:1172-6.

15. Lee SI, Choi YS, Park DJ, Kim HH, Yang HK, Kim MC. Comparative study of laparoscopy-assisted distal gastrectomy and open distal gastrectomy. J Am Coll Surg 2006;202:874-80.

16. Hiki N, Shimoyama S, Yamaguchi H, Kubota K, Kaminishi M. Laparoscopy-assisted pylorus-preserving gastrectomy with quality controlled lymph node dissection in gastric cancer operation. J Am Coll Surg 2006;203:162-9.

17. Ziqiang W, Feng Q, Zhimin C, Miao W, Lian Q, Huaxing L, et al. Comparison of laparoscopically assisted and open radical distal gastrectomy with extended lymphadenectomy for gastric cancer management. Surg Endosc 2006;20:1738-43.

18. Varela JE, Hiyashi M, Nguyen T, Sabio A, Wilson SE, Nguyen NT. Comparison of laparoscopic and open gastrectomy for gastric cancer. Am J Surg 2006;192:837-42.

19. Kim MC, Jung GJ, Kim HH. Learning curve of laparoscopyassisted distal gastrectomy with systemic lymphadenectomy for early gastric cancer. World J Gastroenterol 2005;11:7508-11.

20. Han HS, Kim YW, Yi NJ, Fleischer GD. Laparoscopy-assisted D2 subtotal gastrectomy in early gastric cancer. Surg Laparosc Endosc Percutan Tech 2003;13:361-5. 